\title{
Microwave-Accelerated and Metal-Enhanced Fluorescence Myoglobin Detection on Silvered Surfaces: Potential Application to Myocardial Infarction Diagnosis
}

\author{
Kadir Aslan • Chris D. Geddes
}

Recieved: 28 December 2005 / Accepted: 4 January 2006 / Published online: 1 March 2006

(C) Springer Science+Business Media, Inc. 2006

\begin{abstract}
In this short paper, we describe a novel approach to both significantly accelerate and optically amplify fluorescence-based immunoassays. Our approach utilizes metal-enhanced fluorescence (MEF) to intrinsically optically amplify fluorescence signatures, which, when combined with the use of lowpower microwaves to kinetically accelerate assays, provides for both ultrafast and ultrabright immunoassays. Surprisingly, the use of low-power microwaves and silver nanostructures provides for localized heating, concentrating the effect to the particles themselves as compared to the generic heating of the high dielectric assay fluid. We have subsequently applied our microwave-accelerated MEF approach to the detection of myoglobin, where its rapid quantification is paramount for the clinical assessment of an acute myocardial infarction.
\end{abstract}

Keywords Metal-enhanced fluorescence .

Radiative decay engineering .

Surface-enhanced fluorescence.

Low-power microwaves - Immunoassays .

Ultrasensitive and ultrafast clinical assays · Myoglobin .

Myocardial infarction

K. Aslan · C. D. Geddes $(\bowtie)$

Laboratory for Advanced Medical Plasmonics,

Medical Biotechnology Center,

Institute of Fluorescence,

University of Maryland Biotechnology Institute,

725 West Lombard St., Baltimore, MD 21201, USA

e-mail: Geddes@umbi.umd.edu

C. D. Geddes

Center for Fluorescence Spectroscopy,

University of Maryland School of Medicine,

725 West Lombard St., Baltimore, MD 21201, USA

\author{
Abbreviations \\ AMI acute myocardial infarction \\ SiFs silver-island films \\ SEF surface-enhanced fluorescence \\ MAMEF microwave-accelerated metal-enhanced \\ fluorescence \\ MEF metal-enhanced fluorescence \\ Mw low-power microwaves \\ Myo myoglobin \\ RDE radiative decay engineering
}

\section{Introduction}

Cardiovascular diseases are among the leading causes of mortality in developed countries. It is widely recognized that several cardiac markers should be used for the assessment of a myocardial infarction, such as myoglobin, troponin I, troponin T, and CK-MB [1-3]. While not cardiac specific, myoglobin is one of the very early markers that increases in concentration directly after an acute myocardial infarction (AMI) [4-8] and is therefore a suitable marker for rapid diagnosis using our new microwave-accelerated metalenhanced fluorescence (MAMEF) approach, which offers an ultrarapid and sensitive platform for immunoassays $[9,10]$.

There still remains some debate in the literature today about the value distinguishing between a normal and elevated biomarker level [1-8], with the different markers being present at different levels both before and after the onset of an AMI. For myoglobin, the cutoff value is approximately $100 \mathrm{ng} / \mathrm{ml}$ [11], although some reports of even higher cutoff levels of $200 \mathrm{ng} / \mathrm{ml}$ have been reported [12,13]. Higher limits for myoglobin are often considered because of the fact that 
myoglobin is less specific as compared to other cardiac markers, its level known to fluctuate and be elevated, not only after an AMI, but also because of other factors such as muscular trauma or renal disorder [11].

In a recent paper by our laboratory [9], we have demonstrated MAMEF on a model protein system. MAMEF couples the benefits of MEF, such as increased signal intensities [14-20] and enhanced fluorophore photostabilities [14-20], with the use of low-power microwaves to kinetically accelerate assays and immunoassays $[9,10]$. This recent finding has profound implications and applications in clinical sense. First, the MEF phenomenon allows for much more sensitive assays to be developed, as demonstrated by our laboratories on many occasions [14,16,17], and second, the combination of low-power microwaves to kinetically accelerate assays significantly reduces an immunoassays' run time. Therefore, MAMEF provides for ultrafast and ultrabright immunoassays to be realized.

There are a few medical conditions where the results of an immunoassay are required instantly, such as the diagnosis of an AMI. In a typical hospital setting, a myoglobin immunoassay is usually run on a serum (after blood separation) and can take $>15 \mathrm{~min}$ to kinetically run, the entire cardiac marker screening process taking anywhere from 30 to $60 \mathrm{~min}$ [1-8]. Subsequently, in this short paper, we show how the MAMEF approach can be applied to the rapid and sensitive detection of myoglobin within $20 \mathrm{~s}$, potentially even in whole blood. The MAMEF assay shown here is $>10$ times more sensitive and $>90$-fold more rapid than an identical control assay run on a glass microscope slide and one that is typically employed in clinical settings. In addition, the signal-to-noise ratio of our immunoassay system is $\approx 100$-fold greater than background at $100 \mathrm{ng} / \mathrm{ml}$ myoglobin, which is the typical clinical cutoff level for the assessment of an AMI.

\section{Experimental}

Myoglobin immunoassays were performed in a sandwich format as described previously, with a few modifications [21], and as shown in Figure 1. In this regard, slides were noncovalently coated with a capture anti-myoglobin antibody at room temperature. The glass/silver-island films (SiFs) surfaces were blocked with bovine serum albumin to minimize the nonspecific interaction of the antibodies and myoglobin with the surfaces. These surfaces were then incubated with myoglobin antigen $(100 \mathrm{ng} / \mathrm{ml})$ at room temperature (the clinical cutoff level for myoglobin $[12,13])$ and then used for endpoint measurements. The endpoint measurements were performed by incubating the antigen-coated surfaces in a solution of Alexa 647-labeled anti-myoglobin antibody for $30 \mathrm{~min}$ at room temperature or by microwaving the antigencoated surfaces with Alexa 647-labeled anti-myoglobin antibody for $20 \mathrm{~s}$. Fluorescence measurements were performed by collecting the emission intensity through a long pass filter perpendicular to the assay surface, after total-internal reflection evanescent wave excitation [21], using a 650-nm diode laser and a Fiber Optic Spectrometer (HD2000) from Ocean Optics, Inc. (Figure 2). A detailed description of the microwave cavity and its temperature calibration and the use of a "black body" to control (tune) surface microwave power and therefore the degree of heating has been published elsewhere [9].
Figure 1. Microwave-accelerated metal-enhanced fluorescence myoglobin immunoassay.

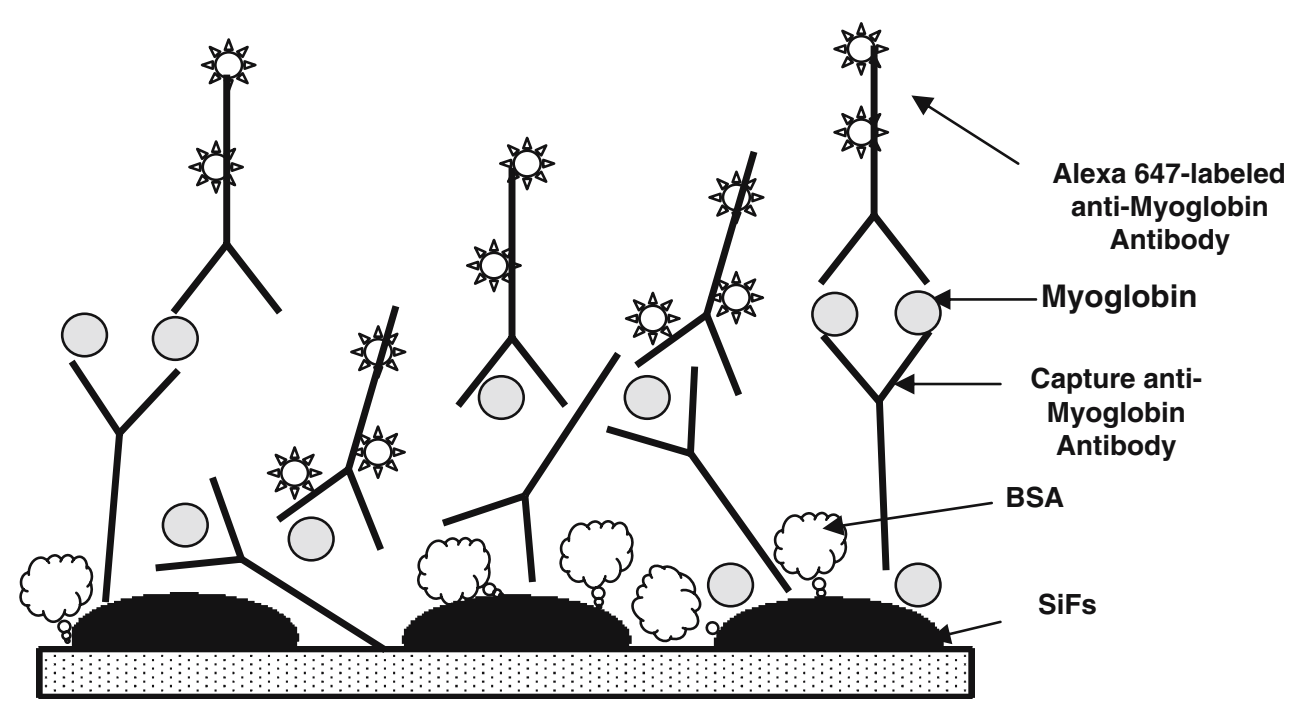




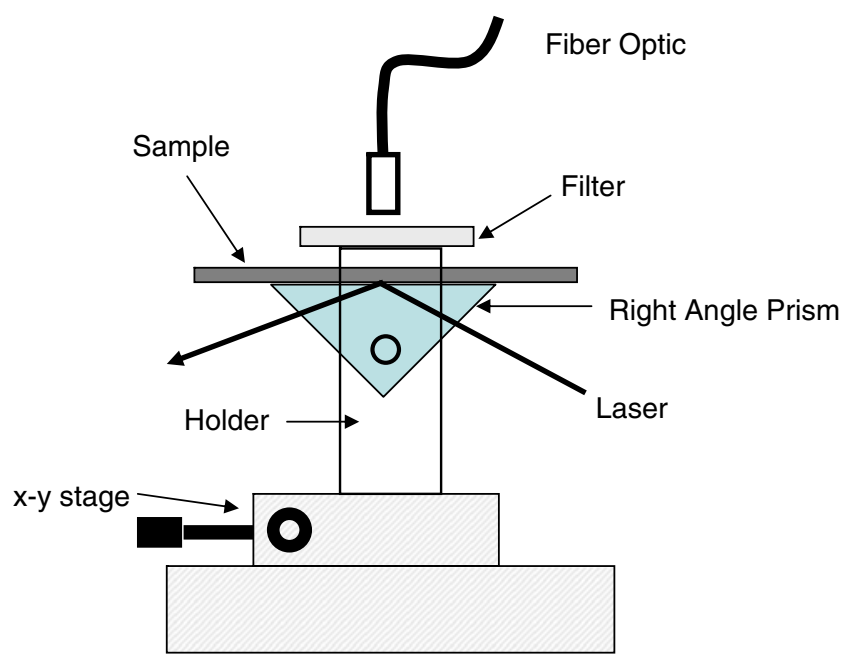

Figure 2. Total-internal reflection fluorescence geometry for myoglobin assessment.

\section{Results and discussion}

Figure 3 shows the plasmon absorption spectra of SiFs (dry), both before and after low-power microwave heating for $30 \mathrm{~s}$. The microwave cavity power was $\approx 140 \mathrm{~W}$, which is the same as used in the myoglobin assay discussed later, and is of a similar power used by others for immunostaining [22,23]. Figure 3 shows that the microwaves and heating had no effect on the surface plasmon absorption of the $\mathrm{SiFs}$, indicating no structural or surface-silver shape changes, where the surface plasmon absorption is well known to be characteristic of the shape of the nanoparticles $[24,25]$. In addition, no sparking was evident from the silvered surfaces, a known consequence of surface charge buildup and dissipation for large nonwavelength-sized particles or continuous surfaces [26]. This

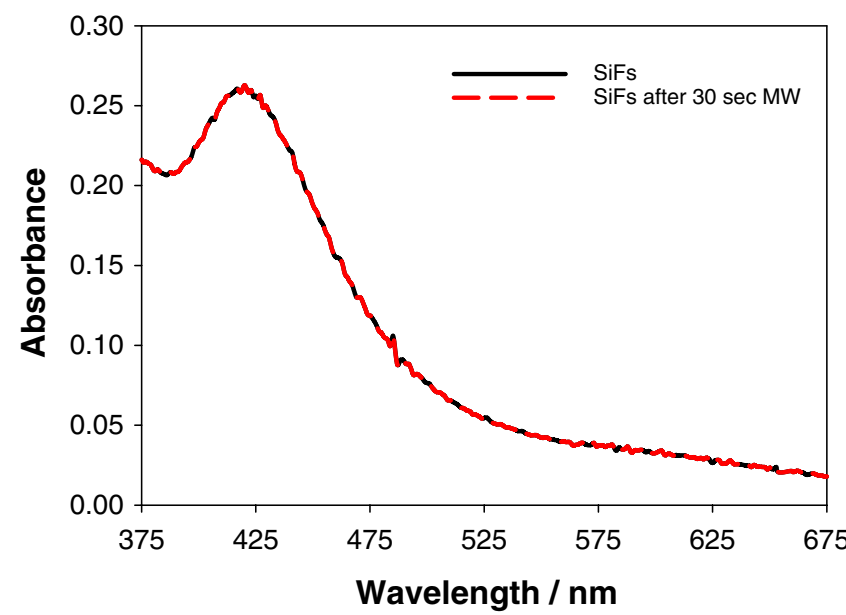

Figure 3. Absorption spectrum of silver-island films (SiFs) both before and after exposure to low-power microwaves. finding is consistent with other findings by our laboratories for silver nanostructures in $2.45-\mathrm{GHz}$ microwave cavities $[9,10]$.

To demonstrate the utility and clinical relevance of our new MAMEF approach for myoglobin detection, we constructed a two-stage assay as shown in Figure 1. First, the assay surface is prepared on both glass and silvered glass substrates, where the glass substrate is used as a control sample by which to compare the usefulness of the silver nanostructures. Myoglobin (100 $\mathrm{ng} / \mathrm{ml}$ ) was subsequently washed over the assay surface followed by incubation of the anti-myoglobin antibody labeled Alexa 647 fluorophore. Figure 4 shows the emission spectra of the labeled Alexa 647 from both the glass and silvered glass regions of the slide, after the anti-myoglobin had been incubated at room temperature for $30 \mathrm{~min}$. A careful investigation of the system has shown that the assay was $\approx 95 \%$ kinetically
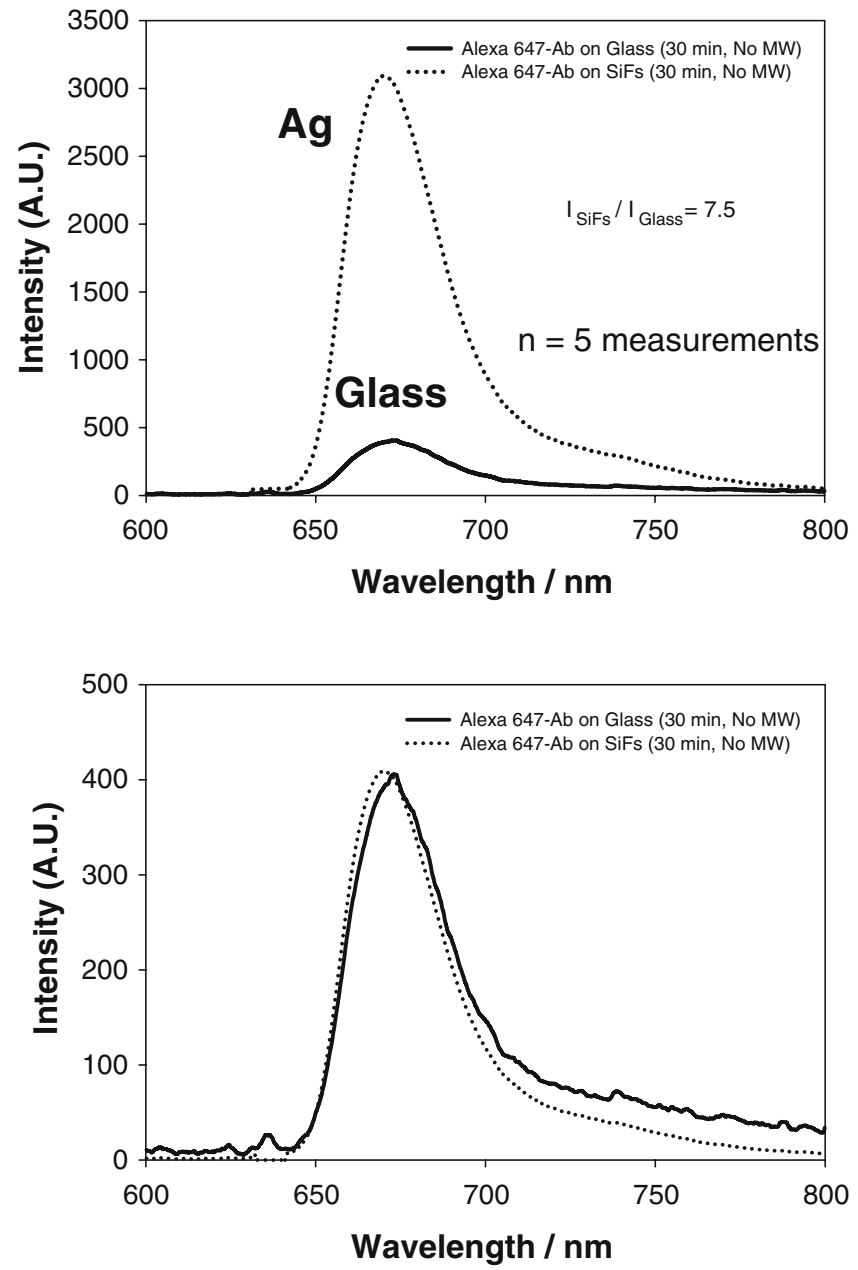

Figure 4. Emission spectrum of Alexa 647 on both SiFs and glass after 30-min incubation (top) and normalized to the fluorescence emission on glass (bottom). The spectra are the mean of five measurements on the respective surfaces. 
complete after 30 -min incubation at $20^{\circ} \mathrm{C}$. From Figure 4 (top), we can see an $\approx 7.5$-fold increase in Alexa 647 emission on the silvered surface, as compared to the glass control, where the spectra shown are the mean of five separate surface measurements. It should be noted that both the glass and silvered portions of the slide had an identical surface coverage of the capture anti-myoglobin antibody as has been shown by our laboratories with various different protein systems [14-20]. Figure 4 (bottom) shows the normalized spectra, demonstrating that the spectral properties are preserved on both the silver and glass substrates.

Figure 5 (top) shows the combined effect of both low-power microwave heating and the optical amplification due to silver for an identical assay as shown in Figure 4. Interestingly, the assay yields an almost identical final emission intensity $(\approx 3000 \mathrm{au})$ after 20 -s microwave heating, as compared to a $30-\mathrm{min}$ room
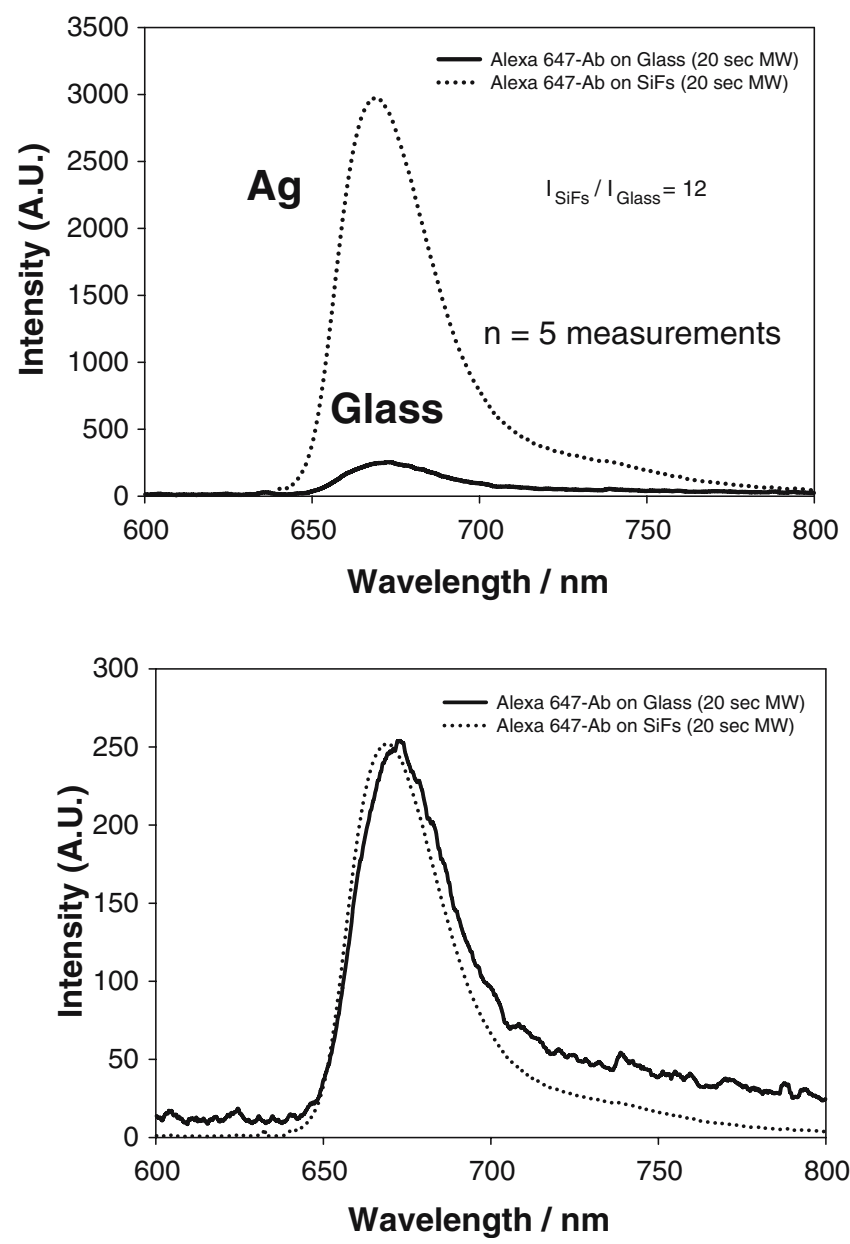

Figure 5. Emission spectrum of Alexa 647 on both $\mathrm{SiFs}$ and glass after 20-s low-power microwave heating (top) and normalized to the fluorescence emission on glass (bottom). The spectra are the mean of five measurements on the respective surfaces. temperature incubation (cf. Figure 4, top). In addition, the silver still maintains its properties for optically enhancing the Alexa 647 fluorescence because of an intrinsic system radiative rate modification [27]. Figure 5 (bottom) shows again that the properties of the Alexa 647 are maintained. This result clearly demonstrates the applicability of low-power microwaves to kinetically accelerate assays, coupled with MEF, to optically enhance fluorescence signatures. In this immunoassay, the use of $\mathrm{SiFs}$ and MAMEF provides for a 12-fold increase in signal, which can be translated to an increased 12 -fold assay sensitivity (i.e., a 12-fold potentially lower myoglobin concentration detection limit), whereas the use of microwaves to facilitate mass protein transport to the surface provides for an $\approx 90$-fold decrease in the assay run time.

A close inspection and comparison of Figures 4 and 5 (top) reveals that the rapidity of the assay is not equal on both the glass and silver substrates. After 30min incubation (Figure 4, top), the glass assay has a maximum emission intensity of about 400 au at 675 nm. In comparison, after 20-s microwave heating (Figure 5, top), the emission intensity on the glass control is $<400 \mathrm{au}$. This trend has also been observed in other recent MAMEF papers by us $[9,10]$. Whereas this decrease lends itself to a larger enhancement ratio after microwave heating, i.e., 12 vs. 7.5 , we believe that this effect is a result of the preferential local heating around the silver nanostructures [26], rapidly accelerating mass transport from the solution to the surface. Our detailed recent microwave calibration studies [9] have shown that, under the conditions employed here, only an $\approx 8^{\circ} \mathrm{C}$ bulk assay temperature jump occurred, which cannot account for the 90-fold increase in assay rapidity, further supporting our notion of localized heating. In addition, others have reported the rapid heating of metallic particles in microwave fields, supporting our observations [26].

\section{Nonspecific assay absorption}

To ascertain the role of microwaves on the extent of nonspecific assay absorption, we undertook several control experiments (Figure 6).

From Figure 6 (top), we can see the emission intensity of the complete assay after only 30 -s incubation, with no microwave heating $(100 \mathrm{ng} / \mathrm{ml}$ myoglobin). Interestingly, the total emission intensity is quite low, as compared to 20-s microwave heating (Figure 5, top). When the assay is incubated with Alexa 647 anti-myoglobin but with no myoglobin in the sandwich immunoassay, then little, if any, nonspecific absorption could be observed (Figure 6, middle). Furthermore, 

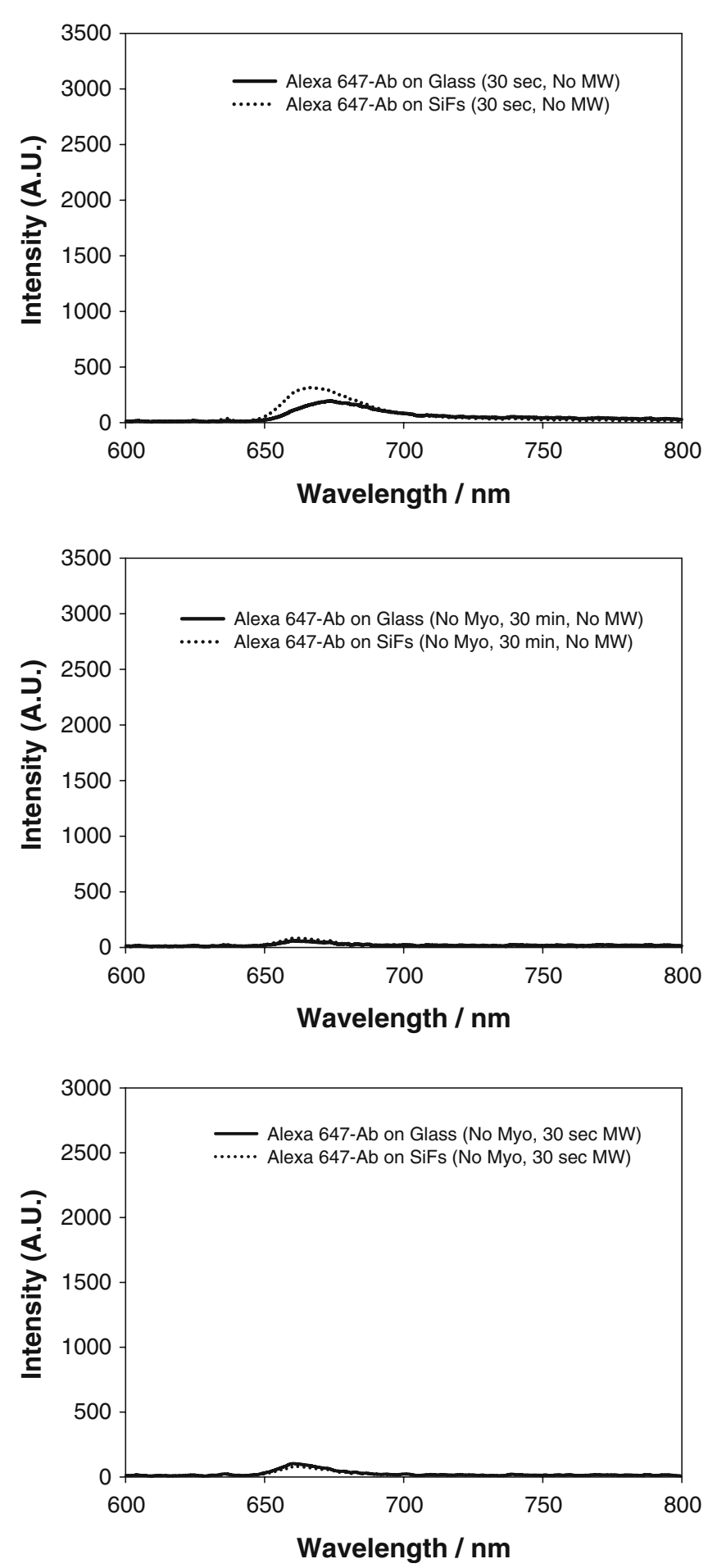

Figure 6. Control experiments: emission spectrum of Alexa 647 on both SiFs and glass after 30 -s incubation at room temperature (with myoglobin; top), after 30-min incubation at room temperature (no myoglobin; middle), and after 30-s low-power microwave heating (no myoglobin; bottom).

after 30-s microwave heating of the assay (with no myoglobin), only a very small amount of nonspecific absorption could be observed $(<100 \mathrm{au}$; Figure 6, bottom). Therefore, we estimate that the contribution of nonspecific absorption toward the total fluorescence emission after 20 -s microwave heating is less than $3 \%$ (cf. Figure 5, top and Figure 6, bottom).

Assay/fluorophore photostability

Fluorophore or analyte photostability is a primary concern in many applications of fluorescence spectroscopy, particularly in platform assays and in single molecule studies $[28,29]$. In our recent reports, we have shown that a reduced fluorescence lifetime of fluorescent species in close proximity to silver nanostructures affords for an increased fluorophore photostability [14-20]. Subsequently, we investigated the combined effect of both constant exposure to 650-nm laser light $(<1 \mathrm{~mW})$ and low-power microwaves on the fluorophore emission. Figure 7 shows the emission intensity value of Alexa 647 from a 100-ng/ml myoglobin immunoassay, which was first microwave-heated to completion for $30 \mathrm{~s}$. The emission from the silvered surface is at least six times greater than on glass. After cumulative and continued exposure to microwaves, the emission intensities on both glass and silver can be seen to be constant, indicating that low-power microwaves do not perturb the fluorophore. It should be noted that the assay volume on the surface was $50 \mu \mathrm{l}$, which did not dry during the microwave heating. A detailed description of the microwave procedure and cavity calibration can be found elsewhere [9].

Benefits of the MAMEF platform in clinical sensing

In this short paper, we have shown an approach for the fabrication of low-cost ultrafast and ultrarapid clinical assays. This new approach addresses two of the main

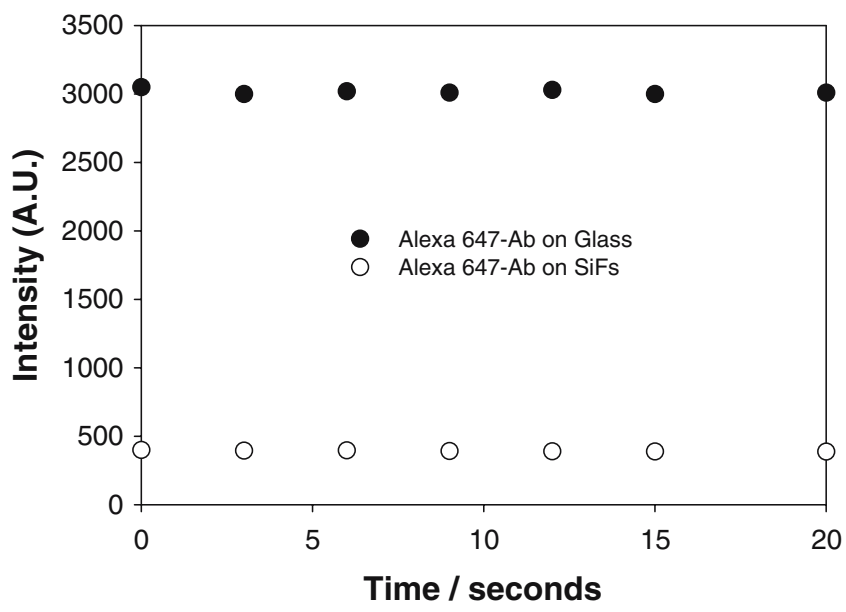

Figure 7. Emission intensity of Alexa 647 on both SiFs and glass after cumulative low-power microwave heating. $\lambda_{\mathrm{ex}}=650 \mathrm{~nm}$. 
bottlenecks with current immunoassays, namely, assay rapidity and sensitivity. Our new MAMEF myoglobin immunoassay has several advantages including the following:

- The MEF phenomenon has been shown to enhance fluorescence signatures up to several thousand folds [30]. Hence, high sensitivity detection for troponins I and $\mathrm{T}$ can readily be envisaged, which have clinical cutoff values $\approx 100$-fold lower than for myoglobin.

- A whole variety of silvered surfaces can be readily prepared [14-20] and also on a variety of substrates, such as plastics [31].

- The evanescent wave mode of excitation used in this paper strongly suggests that the assays can be used in whole blood without the need for serum separation, i.e., enhanced localized excitation above the silver. In this regard, our group has recently reported the detection of biospecies in whole blood using silveramplified evanescent wave excitation [21].

- The low-power microwaves used here do not perturb the silver nanostructures and do not produce arching, which is commonly observed for metallic objects in microwave cavities [26].

- Low-power microwaves substantially increase the rapidity of the assays but not denature the assay proteins, as shown in a recent paper by our group [9].

\section{Conclusions}

In this paper, we have demonstrated a model myoglobin immunoassay for the potential rapid and sensitive detection of a myocardial infarction. The assay demonstrated can readily detect $100 \mathrm{ng} / \mathrm{ml}$ of myoglobin in $20 \mathrm{~s}$, from only $50 \mu \mathrm{l}$ of fluid. The signal-to-noise ratio of the assay is about 100 times above the background for $100 \mathrm{ng} / \mathrm{ml}$ myoglobin, which suggests that this approach could equally be used to detect other more specific cardiac markers, such as either troponin I or T, which have clinical cutoff levels of only a few nanograms per milliliter. Further studies are underway in this regard and will be reported in due course.

Finally, given the low cost and simplicity of the MAMEF technology, coupled with the fact that it potentially provides a generic platform for sensing both clinical analytes and bioterrorism agents, we then envisage that this technology will eventually find general use in the form of disposable one-time use devices for: (1) future home tests kits for clinical assessment and safeguard, such as for myocardial infarction and the related self-diagnosis of chest pains; (2) use by ambu- lance or care workers for the rapid and sensitive diagnosis of various conditions; and (3) a disposable field-use MAMEF test stick utilized by first responders, where the rapid, sensitive, and specific detection of bioagents is of immense national importance.

Acknowledgments This work was supported by the NIH GM070929 and the National Center for Research Resources, RR008119. Partial salary support to CDG from UMBI/MBC is also acknowledged. We also thank Dr. Evgenia Matveeva at the Center for Fluorescence Spectroscopy for help with the preparation of the immunoassays.

\section{References}

1. Ellenius J, Groth T, Lindahl B, Wallentin L (1997) Early assessment of patients with suspected acute myocardial infarction by biochemical monitoring and neural network analysis. Clin Chem 43:1919-1925

2. Newby LK, Storrow AB, Gibler WB, Garvey JL, Tucker JF, Kaplan AL, Schreiber DH, Tuttle RH, McNulty SE, Ohman EM (2001) Bedside multimarker testing for risk stratification in chest pain units-the chest pain evaluation by creatine kinase-MB, myoglobin, and troponin I (CHECKMATE) study. Circulation 103:1832-1837

3. Storrow AB, Gibler WB (1999) The role of cardiac markers in the emergency department. Clin Chim Acta 284:187196

4. Panteghini M, Apple FS, Christenson RH, Dati F, Mair J, Wu AH (1999) Use of biochemical markers in acute coronary syndromes. Clin Chem Lab Med 37:687-693

5. Woo J, Lacbawan FL, Sunheimer R, LeFever D, McCabe JB (1995) Is myoglobin useful in the diagnosis of acute myocardial-infarction in the emergency department setting. Am J Clin Pathol 103:725-729

6. Tucker JF, Collins RA, Anderson AJ, Hess M, Farley IM, Hagemann DA, Harkins HJ, Zwicke D (1994) Value of serial myoglobin levels in the early diagnosis of patients admitted for acute myocardial-infarction. Ann Emerg Med 24:704-708

7. Montague C, Kircher T (1995) Myoglobin in the early evaluation of acute chest pain. Am J Clin Pathol 104:472476

8. Kilpatrick WS, Wosornu D, McGuinness JB, Glen AC (1993) Early diagnosis of acute myocardial-infarctionck-mb and myoglobin compared. Ann Clin Biochem 30:435-438

9. Aslan K, Geddes CD (2005) Microwave-accelerated metalenhanced fluorescence (MAMEF): a new platform technology for ultra-fast and ultra-bright assays. Anal Chem 77(24):8057-8067

10. Aslan K, Geddes CD (in press) Microwave-accelerated metal-enhanced fluorescence (MAMEF): application to fast and sensitive clinical assays. J Fluoresc

11. Matveeva E, Gryczynski Z, Gryczynski I, Malicka J, Lakowicz JR (2004) Myoglobin immunoassay utilizing directional surface plasmon-coupled emission. Anal Chem 76, 6287-6292

12. Castaldo AM, Ercolini P, Forino F, Basevi A, Vrenna L, Castaldo P, Ambrosio VD, Castaldo A (1994) Eur J Clin Chem Clin Biochem 32(5), 349-353 
13. Hillis GS, Zhao N, Taggart P, Dalsey WC, Mangione A (1999) Utility of cardiac troponin I, creatine kinase-MBmass, myosin light chain 1 , and myoglobin in the early in-hospital triage of "high risk" patients with chest pain. Heart 82(5): 614-620

14. Aslan K, Gryczynski I, Malicka J, Matveeva E, Lakowicz JR, Geddes CD (2005) Metal-enhanced fluorescence: an emerging tool in biotechnology. Curr Opin Biotechnol 16(1):55-62

15. Geddes CD, Lakowicz JR (2002) Metal-enhanced fluorescence. J Fluoresc 12(2):121-129

16. Geddes CD, Aslan K, Gryczynski I, Malicka J, Lakowicz JR (2005) In: Geddes CD, Lakowicz JR (eds) Topics in Fluorescence in Fluorescence Spectroscopy, Kluwer Academic/Plenum Publishers, New York, USA, pp 401-448

17. Geddes CD, Aslan K, Gryczynski I, Malicka J, Lakowicz JR (2004) In: Geddes CD, Lakowicz JR (eds) Review Chapter for Annual Reviews in Fluorescence 2004, Kluwer Academic/Plenum Publishers, New York, USA, pp 365-401

18. Aslan K, Lakowicz JR, Geddes CD (2005) Rapid deposition of triangular silver nanoplates on planar surfaces: application to metal-enhanced fluorescence. J Phys Chem B. 107(13):6247-6251

19. Aslan K, Leonenko Z, Lakowicz JR, Geddes CD, (2005) Fast and slow deposition of silver nanorods on planar surfaces: application to metal-enhanced fluorescence. J Phys Chem B 109(8):3157-3162

20. Geddes CD, Parfenov A, Roll D, Fang J, Lakowicz JR (2003) Electrochemical and laser deposition of silver for use in metal-enhanced fluorescence. Langmuir 19(15):62366241
21. Matveeva E, Gryczynski Z, Lakowicz JR, (2005) Myoglobin immunoassay based on metal particle-enhanced fluorescence. J Immunol Methods 302:26-35

22. Micheva KD, Holz RW, Smith SJ (2001) Regulation of presynaptic phosphatidylinositol 4,5-biphosphate by neuronal activity. J Cell Biol 154:355-368

23. Petrali JP, Mills KR (1998) Microanalysis 4:114-115

24. Link S, El-Sayed MA (1999) Spectral properties and relaxation dynamics of surface plasmon electronic oscillations in gold and silver nanodots and nanorods. J Phys Chem B 103:8410-8426

25. Kreibig U, Genzel L (1985) Surf Sci 156:678-700

26. Whittaker AG, Mingos DMP (1993) Microwave-assisted solid-state reactions involving metal powders and gases. J Chem Soc Dalton Trans 16:2541-2543

27. Aslan K, Leonenko Z, Lakowicz JR, Geddes CD (2005) Annealed silver island films for metal-enhanced fluorescence. J Fluoresc 15(5):643-654

28. Lakowicz JR (1999). Principles of Fluorescence Spectroscopy. Kluwer, New York

29. Axelrod D, Hellen EH, Fulbright RM (1992) Total internal reflection fluorescence. In: Lakowicz JR (ed) In Topics in Fluorescence Spectroscopy, Vol. 3: Biochemical Applications. Plenum Press, New York, pp 289-343

30. Parfenov A, Gryczynski I, Malicka J, Geddes CD, Lakowicz JR (2003) Enhanced fluorescence from fluorophores on fractal silver surfaces. J Phys Chem B 107(34):8829-8833

31. Aslan K, Badugu R, Lakowicz JR, Geddes CD (2005) Metalenhanced fluorescence from plastic substrates. J Fluoresc 15(2):99-104 